\title{
Chemical Shift Magnetization Transfer Magnetic Resonance Imaging
}

\author{
Weiguo Li ${ }^{1}$, Xifu Wang ${ }^{1}$, Frank H. Miller ${ }^{1}$, and Andrew C. Larson ${ }^{1}$ \\ ${ }^{1}$ Department of Radiology, Northwestern University, Chicago, Illinois, USA
}

\section{Abstract \\ Purpose-To develop a chemical shift magnetization transfer (CSMT) MRI method to provide accurate MTR measurements in the presence of fat.}

\begin{abstract}
Methods-Numerical simulations were performed to compare MTR measurements at different echo-times (TEs) for voxels with varying fat/water content. The CSMT approach was developed using water fraction estimates to correct for the impact of fat signal upon observed MTR measurements. CSMT method was validated with oil/agarose phantom and animal studies.
\end{abstract}

\begin{abstract}
Results-Simulations demonstrated that the observed MTRs vary with water fraction as well as with the TE dependent phase difference between fat and water signals; simulations also showed that a linear relationship exists between MTR and water fraction when fat and water signals are inphase. For phantom studies, observed MTR decreased with increasing oil fraction: $42.41 \pm 0.54$, $38.12 \pm 0.33,32.93 \pm 0.56$ and $26.08 \pm 0.87$ for $5 \%$ to $40 \%$ oil fractions respectively, compared to $42.63 \pm 1.04$ for phantom containing $4 \%$ agarose only. These offsets were readily corrected with the additional acquisition of a water fraction map.
\end{abstract}

Conclusion-Fat fraction and TE can significantly impact observed MTR measurements. The new CSMT approach offers the potential to eliminate the effects of fat upon MTR measurements.

\section{Keywords}

Magnetization transfer; fat; chemical shift; fat water separation

\section{Introduction}

Magnetization transfer (MT) is a dynamic process that involves the exchange of magnetization between sub-populations of free water protons and those water protons bound to macromolecules (e.g., proteins and phospholipids)(1). The composition of macromolecules can vary widely between different tissues and disease states. MR pulse sequences can be sensitized to the MT phenomenon thus producing images with marked signal contrast between different tissue types. MT MRI has been widely used for the study of neurological diseases, such as multiple sclerosis (2) and Alzheimer's disease (3) and more recently for in vivo assessments of tissue fibrogenesis in animal models of cancer (4) and

Please send proof and correspondence to: Weiguo Li, Department of Radiology, Feinberg School of Medicine, Northwestern University, 737 N. Michigan Ave, 16th Floor, Chicago, IL 60611, Phone: (312)926-3499, Fax: (312)926-5991, Weiguo- 
Crohn's disease (5). However, application of MT MRI approaches for diagnostic abdominal imaging has been limited, particularly for examination of the liver. Accurate differentiation of healthy and fibrotic tissues (6-8) with conventional MT MRI methods may be difficult due to the common existence of fat within abdominal organs.

An effective fat elimination technique should increase the precision of MT measurements. Fat-suppression and water- or fat-selective imaging have been used in many routine clinical examinations to reduce the so-called chemical shift artifacts for improved tissue characterization $(9,10)$. However, fat-suppression and water- or fat-selective pulses may cause additional MT effects and/or alter the steady-state of MT sequences. Furthermore, these methods can be sensitive to both static field $\left(\mathrm{B}_{0}\right)$ and/or radiofrequency field $\left(\mathrm{B}_{1}\right)$ heterogeneity. It has been reported that MT saturation does not affect fat signal due to the absence of an MT pathway from fat to water or from fat to protein and membrane phospholipid protons (11). A fat-free MR image sensitive to MT in fatty tissues was previously reported (12) and most recently, MT ratio (MTR), the overall description of the MT phenomenon, was found to vary according to pulse sequence echo time and water fraction in the presence of fat $(13,14)$. In addition, MT-weighted images of human optical nerve were found less affected by the surrounding tissues (e.g., orbital fat and meninges) when corrected with a water image derived from three-point Dixon method (15). The latter studies suggest that a systematic investigation of fat effects upon MTR measurements is clearly warranted. A technique that can eliminate the effect of fat signal interference should expand applications of MT MRI to a broad range of diseases that can include simultaneous fat deposition and pathologic changes in macromolecular content, for example, nonalcoholic steatohepatitis (16), fibrocystic breast changes (17), Crohn's disease (18), and Polycystic Ovarian Syndrome (19).

In this study, we explore the mechanism of how fat signal can impact MTR measurements and develop a new chemical shift magnetization transfer (CSMT) MRI technique that combines chemical shift based fat/water separation and magnetization transfer saturation techniques to provide accurate MTR measurements even in the presence of fat. Numerical, phantom, and animal studies were performed to demonstrate the feasibility of the newly proposed method.

\section{Theory}

The MRI signal from a voxel at the presence of fatty tissue, free water, and macromolecules includes the signals from both water and fat. While neglecting the contribution from relaxation, the MRI signal can be expressed as

$$
\mathbf{M}=\alpha \mathrm{M}_{\mathrm{a}}+(1-\alpha) \mathrm{M}_{\mathrm{a}} e^{j \cdot 2 \pi \cdot \Delta f \cdot T E} \quad(\text { Eq. 1) }
$$

$\mathbf{M}$ is the total MRI signal in complex form. $\mathrm{M}_{\mathrm{a}}$ is the signal from the total protons from both free water and fatty tissue. $a$ is the water proton fraction. $\Delta f$ is the chemical shift between water and fat. TE is the echo time. 
Since water does not exchange magnetization with triglyceride or fat protons in the tissue (11), the observed MTR (MTR ${ }_{\text {obs }}$ ) can be expressed as

$M T R_{\mathrm{obs}}=100 \times \frac{\left|\alpha \mathrm{M}_{\mathrm{a}}+(1-\alpha) \mathrm{M}_{\mathrm{a}} e^{j \cdot 2 \pi \cdot \Delta f \cdot T E}\right|-\left|\left(\alpha \mathrm{M}_{\mathrm{a}}\right)_{s a t}+(1-\alpha) \mathrm{M}_{\mathrm{a}} e^{j \cdot 2 \pi \cdot \Delta f \cdot T E}\right|}{\left|\alpha \mathrm{M}_{\mathrm{a}}+(1-\alpha) \mathrm{M}_{\mathrm{a}} e^{j \cdot 2 \pi \cdot \Delta f \cdot T E}\right|}$

$\left(\mathrm{aM}_{\mathrm{asat}}\right.$ represents the signal contributed via MT from macromolecules to the water fraction following MT saturation. Eq. 2 indicates MTR $_{\text {obs }}$ changes with both TE and a.

While water and fat are in phase, $\mathrm{MTR}_{\mathrm{obs}}$ becomes

$$
M T R_{\mathrm{obs}}=100 \times \frac{\mathrm{M}_{w}-\mathrm{M}_{\mathrm{satw}}}{\mathrm{M}_{\mathrm{a}}}
$$

$\mathrm{M}_{\text {satw }}$ and $\mathrm{M}_{W}$ represent the signal intensity from water with and without MT saturation, respectively. We define the true MTR $\left(\mathrm{MTR}_{\text {true }}\right)$ as the MTR value without the interference of fat, that is,

$$
M T R_{\text {true }}=100 \times \frac{\mathrm{M}_{w}-\mathrm{M}_{\text {satw }}}{\mathrm{M}_{w}} \quad \text { (Eq. 4) }
$$

Thus, from Eq. 3 and 4, MTR true becomes

$$
M T R_{\text {true }}=1 / \alpha \cdot M T R_{\text {obs }} \quad \text { (Eq. 5) }
$$

Eq. 5 indicates that a correction factor, $\mathrm{a}$, can be applied to $\mathrm{MTR}_{o b s}$ to generate $\mathrm{MTR}_{\text {true }}$ when fat and water signals are in-phase. With the assumption of an equal relative proton density between water and fat, one can approximate a for each voxel based upon corresponding water signal fraction measurements performed using chemical shift based fat/ water separation techniques (20-22).

\section{Methods}

\section{Simulation}

Numerical simulations were performed using Matlab software (MathWorks, Natick, MA). For demonstration purposes, we plotted Eq. $1 \& 2$ with an assumption of a single MTR true $_{\text {of }}$ of 60 , water-fat chemical shift of $3.5 \mathrm{ppm}$, water fractions ranging from 0 to 1 , and product of $\mathrm{TE}$ and $\mathrm{B}_{0}$ ranging from 0 to $20 \mathrm{~ms} \bullet$ tesla.

\section{Phantom}

Four oil/agar phantoms were constructed with peanut oil (as a mimic of body fat) with volume fractions of $5 \%, 10 \%, 20 \%$, and $40 \%$ and agarose concentrations of $4 \%$ (weight-by- 
volume) respectively. Four fluorocarbon/agar phantoms, for comparison, were composed of a fluorocarbon-based fluid - Fluorinert electrical liquid (3M, MN) with volume fractions of $5 \%, 10 \%, 20 \%$, and $40 \%$ and agarose concentrations of $4 \%$ respectively. In short, $4 \%$ agarose gel was heated to liquid phase. Peanut oil or Fluorinert and the agarose gel were then emulsified manually by pumping the mixture back and forth through a three-way stopcock (Cook, Bloomington, IN) for 2 minutes. The mixture was placed in a $20 \mathrm{~mL}$ scintillation vial (Sigma-Aldrich, St. Louis, MO) within $4^{\circ}$ refrigerator for the gel to form. An additional three $20 \mathrm{~mL}$ scintillation vials were filled with peanut oil, Fluorinert electrical liquid, and $4 \%$ agarose to serve as controls. A $0.03 \mathrm{mM} \mathrm{MnCl}_{2}$ (Sigma-Aldrich, St. Louis, MO) solution was used to evaluate the direct saturation effect of the MT pulse (23).

\section{Phantom MRI}

MRI studies were performed using a 3-T MR scanner and a 12-channel head coil (Magnetom Trio; Siemens, Erlangen, Germany). The $\mathrm{B}_{0}$ field was carefully shimmed prior to each CSMT measurement to migrate potential effects of $\mathrm{B}_{0}$ inhomogeneity. For chemical shift based fat/water separation, a 3D multiple gradient echo (MGRE) pulse sequence was used with the following acquisition parameters: repetition time (TR) of $20 \mathrm{~ms}$, echo time (TE) of 1.19, 2.46, 3.69, 4.92, 6.15, and $7.38 \mathrm{~ms} ; 128 \times 104$ matrix; $140 \times 114 \mathrm{~mm}$ field of view (FOV); $5^{\circ}$ flip angle (FA); $5 \mathrm{~mm}$ section thickness; $1030 \mathrm{~Hz} /$ pixel bandwidth (BW); and 16 averages. MT acquisitions were performed using a single slice two-dimensional spoiled gradient-echo acquisition with following parameters: TR/TE, 36/2.24 ms; FA, $9^{\circ}$; BW, $1030 \mathrm{~Hz} /$ pixel; matrix, $128 \times$ 104; slice thickness, $5 \mathrm{~mm}$; FOV, $140 \times 114 \mathrm{~mm}$; and 16 averages. MT saturation was applied using a Gaussian RF pulse with pulse length of $10 \mathrm{~ms}$, FA of $800^{\circ}$, and 23 off-resonance frequencies from 0 to $100 \mathrm{kHz}$. To evaluate the direct saturation effect and MT saturation power, z-spectra were obtained with 23 offset frequencies from $0 \mathrm{~Hz}$ to $100 \mathrm{kHz}$ and three MT saturation power levels $\left(\mathrm{FA}=400^{\circ}, 600^{\circ}\right.$, and $800^{\circ}$ respectively). One MT acquisition was performed with $\mathrm{TE}=3.36 \mathrm{~ms}$ (all other acquisition parameters remaining the same) to show MT weighted images in which water and fat signals are out of phase.

\section{Animal MRI}

The animal model MRI experiments were carried using a New Zealand white rabbit under a protocol approved by the Institutional Animal Care and Use Committee (IACUC). The rabbit was placed in a 12-channel head coil within same 3-T MR scanner used for phantom studies. The same 3D MGRE sequence was applied for fat/water separation with the acquisition parameters: TR, $20 \mathrm{~ms}$; TE, 1.19, 2.46, 3.69, 4.92, 6.15, and $7.38 \mathrm{~ms}$; FA, $6^{\circ}$; BW, $1040 \mathrm{~Hz} /$ pixel; matrix, $192 \times 150$; FOV, $222 \times 173 \mathrm{~mm}$; slice thickness, $3 \mathrm{~mm}$; and 1 averages. The MT sequence was applied with following parameters: TR/TE, 36/2.24 ms; FA, $9^{\circ}$; BW, $1030 \mathrm{~Hz} /$ pixel; matrix, $128 \times 100$; slice thickness, $3 \mathrm{~mm}$; FOV, $222 \times 173 \mathrm{~mm}$; and 16 averages. MT saturation was applied using a Gaussian RF pulse with pulse length of 10 $\mathrm{ms}$, FA of $600^{\circ}$, and an off-resonance excitation frequency of $1500 \mathrm{~Hz}$.

\section{Data Analysis}

Post-processing was performed offline using Matlab software (MathWorks, Natick, MA). A multi-step adaptive fitting based fat-water separation method was used to extract the voxel- 
wise oil and water volume fraction maps (24). In brief, Levenberg-Marquardt non-linear fitting was used to fit the magnitude of the complex signal of the multi-echo data according to a multi-peak chemical shift model. For each voxel, the water percentage was calculated along with the corresponding $\mathrm{R}_{2} *$ value of the water-fat mixture. To obtain mean and standard deviation (SD) of MTR measurements within each phantom vial, voxel-wise MTR maps were produced and region of interests (ROIs) drawn to at the center of the MTweighted image of each phantom. Once defined within one image, the ROIs were transferred to all corresponding MTR maps at each offset frequencies as well as the corresponding water fraction map. For each ROI, the $\mathrm{MTR}_{\text {true }}$ from each oil/agar phantom was calculated using Eq. 5. The reference MTR (MTR ${ }_{\text {ref }}$ ) was generated from MTR $_{\text {obs }}$ map of each fluorocarbon/agar phantom using ROI drawn at center of the phantom image. Direct saturation effects were evaluated using ROIs drawn within $\mathrm{MnCl}_{2}$ and pure peanut oil phantom $\mathrm{MTR}_{\mathrm{obs}}$ maps. Direct saturation was considered negligible if $\mathrm{MTR}_{\mathrm{obs}}$ was less than 5. The percentage errors for $\mathrm{MTR}_{\mathrm{obs}}$ and $\mathrm{MTR}_{\text {true }}$ measurements relative to $\mathrm{MTR}_{\text {ref }}$ were calculated according to the following two equations: $100 \times\left(\mathrm{MTR}_{\mathrm{obs}}-\mathrm{MTR}_{\mathrm{ref}}\right) / \mathrm{MTR}_{\mathrm{ref}}$ and $100 \times\left(\mathrm{MTR}_{\text {true }}-\mathrm{MTR}_{\text {ref }}\right) / \mathrm{MTR}_{\text {ref }}$.

\section{Results}

Fig. 1 illustrates simulation results demonstrating the influence of water (or fat) fraction and TE upon MTR measurements $\left(M_{T R} R_{\text {obs }}\right)$. Fig. 1a indicates that the normalized MRI signal (M) varies from the minimum of zero, when water fraction is 0.5 and fat and water signal are opposed phase, to the maximum of 1 when fat and water are in phase. Simulated MTR $_{\mathrm{obs}}$ (Fig. 1 b) changes with water fractions as well with changes in TE $\bullet \mathrm{B}_{0}$. Resulting $\mathrm{MTR}_{\mathrm{obs}}$ can be negative when water fraction is smaller than fat fraction and water and fat signals are not in phase (Fig. 1c). When water and fat signal are in opposed phase, the simulated MTRs values varied widely with changes in the water (or fat) fraction (turquoise line in Fig. 1c). $\mathrm{MTR}_{\mathrm{obs}}$ and water fraction were linearly related for those $\mathrm{TE} \cdot \mathrm{B}_{0}$ that resulted in in-phase fat and water signals (black line in Fig. 1c).

The phantom configuration is depicted in Fig. 2a with fluorocarbon/agar phantoms in the left column, oil/agar phantoms in the middle, and the other control phantoms $-0.03 \mathrm{mM}$ $\mathrm{MnCl}_{2}, 4 \%$ agarose, pure peanut oil, and pure Fluorinert electrical liquid — in the right column. The water fraction map for all the phantoms with $4 \%$ agarose is shown in Fig. $2 \mathrm{~b}$. Decreased water fraction was observed for the four oil/agar phantoms with percentages of $94.3 \pm 2.1,88.3 \pm 2.2,79.5 \pm 3.2$, and $61.4 \pm 2.4$ for $5 \%$ to $40 \%$ of oil fractions. As shown in the representative MT weighted images (Fig. 2c), in which fat and water signals are in phase, pure Fluorinert produced no MR signal. Fluorocarbon/agar phantoms showed visibly lower signal intensities compared to the corresponding oil/agar phantoms. When fat and water signals are in phase, MTR $_{\text {obs }}$ for oil/agar phantoms (middle column in Fig. 2d) decreased with increasing oil fraction when compared to $4 \%$ agarose phantom and corresponding fluorocarbon/agar phantoms. $\mathrm{MTR}_{\mathrm{obs}}$ measurements for the four oil/agar phantoms were $42.41 \pm 0.54$ (mean \pm SD), $38.12 \pm 0.33,32.93 \pm 0.56$ and $26.08 \pm 0.87$ for $5 \%$ to $40 \%$ oil fractions respectively. The $4 \%$ agarose gel and fluorocarbon/agar phantoms demonstrated comparable $\mathrm{MTR}_{\mathrm{obs}}$ measurements of $42.48 \pm 0.70$ for $4 \%$ agarose gel, and $43.34 \pm 0.60,41.20 \pm 0.65,41.51 \pm 0.53$, and $42.63 \pm 1.04$ for $5 \%$ to $40 \%$ Fluorinert in 
agarose gel, respectively. With the exception of pure peanut oil, comparable MTRs (shown in Fig. 2e) were obtained for these same oil/agar phantoms once the $\mathrm{MTR}_{\text {obs }}$ map was corrected using the acquired water fraction map (Fig. 2b). Fig. 2f shows an MTR map acquired with fat and water signals out of phase $(\mathrm{TE}=3.36 \mathrm{~ms})$ resulting in $\mathrm{MTR}_{\text {true }}$ measurements of $43.77 \pm 2.54,40.13 \pm 1.51,58.26 \pm 0.71$ and $101.66 \pm 0.73$ for $5 \%$ to $40 \%$ oil fractions respectively, and $43.32 \pm 0.67$ for $4 \%$ agarose gel.

$\mathrm{Z}$ spectra (Fig. S1a [online]) show the direct saturation effect at various offset frequencies with MT saturation powers of $400^{\circ}, 600^{\circ}$ and $800^{\circ}$ respectively. For pure peanut oil, direct saturation was observed for MT saturation frequencies less than $400 \mathrm{~Hz}$ (Fig. S1a [online]) for MT saturation power of $800^{\circ}$. An overall picture for visual comparison of MT effects upon all the phantoms used in this study at the different offset frequencies is shown in Fig. $\mathrm{S} 1 \mathrm{~b}$ [online]. Agarose gels showed higher MT saturation compared to $\mathrm{MnCl}_{2}$ and pure peanut oil at most offset frequencies (Fig. S1b [online]).

Fig. 3 illustrates MTRs at various MT pulse offset frequencies. For each MT pulse frequency offset, the $\mathrm{MTR}_{\mathrm{obs}}$ of agarose gel phantom was found to be greater than that of $\mathrm{MnCl}_{2}$ and pure peanut oil, for which direct saturation largely contributed to the signal changes (Fig. $3 a)$. The direct saturation effect upon the pure peanut oil was less than $2 \%$ with the MT pulse saturation of $800^{\circ}$ and offset frequencies greater than $1000 \mathrm{~Hz}$ (shown in the mezzanine of Fig. 3a). For the four oil/agar phantom compositions (Fig. 3b), MTR $_{\text {obs }}$ decreased as the MT pulse frequency offset increased. Significantly lower MTR $_{\text {obs }}$ values were found for oil/agar phantoms with oil fractions of 10\%, 20\% and 40\% than for agar and fluorocarbon/agar phantoms (Fig. 3b and c). For fluorocarbon/agar phantoms (Fig. 3c), $\mathrm{MTR}_{\mathrm{obs}}$ decreased as the MT pulse frequency offset increased, but similar $\mathrm{MTR}_{\mathrm{obs}}$ to those in agar-only phantoms was observed at all offset frequencies. Corrected MTRs (MTR true) for the oil/agar phantoms (calculated using Eq. 5) were markedly close to those measured for agarose-only and fluorocarbon/agar phantoms (Fig 3d). As indicated in Fig. 4, much smaller percentage errors were found between $\mathrm{MTR}_{\text {true }}$ and $\mathrm{MTR}_{\text {ref }}$ measurements for all compositions of oil/agar phantoms compared to the percentage errors between $\mathrm{MTR}_{\mathrm{obs}}$ and $\mathrm{MTR}_{\text {ref }}$ measurements at the corresponding MT offset frequencies.

The application of the CSMT method on a rabbit model is shown in Fig.5. The opposedphase MR image (Fig. 5a) shows a sharply defined dark rim surrounding many of the visceral organs (the included arrows point to this fat-water interface at the kidney, skeletal muscle, and liver). The corresponding in-phase MR image (Fig. 5b) shows moderately increased susceptibility artifacts in the gas-filled stomach and intestines (arrows) compared with those on the opposed-phase image due to the longer echo time. Visceral and subcutaneous fatty tissues (red arrows) were clearly observed in the water-fraction map (Fig. 5c). The MT-weighted image (Fig. 5d) indicated higher MT saturation effects in the skeletal muscle, liver, and kidney compared to surrounding fatty tissue. The $\mathrm{MTR}_{\text {true }}$ map (Fig. 5f) shows slight increases of MTR values in skeletal muscle, liver, and kidney comparing to the $\mathrm{MTR}_{\text {obs }}$ map (Fig. 5e) because the major component of these organs in this rabbit is water as shown in the water fraction map (Fig. 5c). 


\section{Discussion}

Numerical simulations demonstrated the mechanisms by which fat signal can complicate MTR measurements and a new CSMT method was developed to remove fat interference from these MTR measurements. The CSMT approach exploited the water fraction extracted from chemical shift based fat/water separation techniques to provide a correction factor permitting more accurate MTR measurements in the presence of fat. Using oil/agar phantom models we then demonstrated that the effects of various oil fractions can be significantly reduced using the CSMT approach to generate comparable MTR values to those of corresponding agarose gel-only phantoms (that contained no oil).

The evaluation of MT properties in tissues containing fat can be challenging. MTR measurements are well known to be sensitive to tissue collagen content and fibrosis $(5,25)$. However, MTR measurements have failed to serve as a specific marker of fibrosis in the liver (6-8). Coexistence of fibrotic tissue with inflammation and variable degrees of hepatic steatosis as well as motion artifacts during hepatic imaging were cited as possible causes of the inability of conventional MTR measurement to differentiate healthy and fibrotic livers $(6,7,26)$. In our study, we demonstrated that the observed MTR can vary with changes in both water fraction and TE-dependent phase differences (Eq. 2, Fig. 1 and Fig. 2).

According to these simulation results, there was a wide range for both M (Fig. 1a) as would be expected given phase differences and observed MTRs (Fig. 1b). When fat and water signals were not in phase, MT saturation could actually result in higher magnitude signal intensities if the water fraction was smaller in the voxel, thus leading to a negative observed MTR as shown in Fig. 1b and c. The complexity of observed MTR could offer a potential explanation for the failure of MTR measurements in liver fibrosis applications -fat and water signals were potentially not entirely in-phase according to the different TEs and MT sequences utilized for the studies (6-8, 27-29). However, clearly further in vivo studies in either pre-clinical models or patients will be necessary to rigorously clarify this impact and importantly validate that the proposed CSMT method can overcome prior limitations.

Theoretically, as shown in Eq. 5, CSMT methods can generate MTR measurements without interference from fat signals, thus, potentially helping to mitigate the effect of fat during MTR measurements in diseases such as non-alcoholic steatohepatitis wherein fatty tissue infiltration occurs simultaneously to changes in the macromolecular content (16). However, successful application of this method relies upon four key presumptions: 1) fat protons do not exchange magnetization with free water protons; 2) fat and water signals are in-phase at certain TEs; 3) accurate water and fat fractions can be accurately carried out; and 4) MT direct saturation effects on fatty tissue can be neglected. For this study carried out at 3-Tesla, a TE of $2.24 \mathrm{~ms}$ was chosen for the MT sequence to ensure signals from peanut oil and agarose gel were close to in-phase. Our results (Fig 3b) clearly showed that MTR $_{\text {obs }}$ decreased with the increase in peanut oil content. Additionally, our results indicated that peanut oil was less affected by the direct saturation of MT RF pulse than the $\mathrm{MnCl}_{2}$ solution that usually serves as the controls for tissue direct saturation effects (Fig 3a). The MTRs observed due to the direct saturation in peanut oil were negligibly small $(<2)$ with MT RF pulse power of $800^{\circ}$ and offset frequencies greater than $1000 \mathrm{~Hz}$ (Fig. 3a), indicative of negligible direct saturation effects across the applied range of sequence parameters. By 
using the water fraction extracted from a fat/water separation technique as the correction factor, for those MT saturation powers and offset frequencies with minimal direct saturation on fat tissue, the CSMT method provided consistently accurate MTR measurements across a broad range of MT saturation offset frequencies (Fig. 3d). The small percentage errors observed between MTR $_{\text {true }}$ and those of corresponding controls (Fig. 4b) indicated our CSMT approach can effectively eliminate the interference of fat upon the MTR measurements. Additionally, the rabbit model results (Fig. 5) demonstrated the feasibility of our method for future in vivo and clinical studies. We observed higher MTR values for MTR $_{\text {true }}$ versus MTR $_{\text {obs }}$ in muscle, liver, and kidney tissues with latter results likely due the large water fraction within these organs.

For ideal MTR measurements, it would be preferable to use images with only MT contrast. However, in reality, it is almost impossible to have MR images from solely one contrast mechanism. Therefore, the water-only images extracted from the fat/water separation techniques that reflect signals from the proton density in the water fraction could be more straightforward for the MTR calculation. A potential alternative approach to our proposed method would be to individually solve for water fraction images at each offset saturation frequency (following acquisition of MT-weighted MGRE images for fat/water separation at each offset saturation frequency). Smith and Dorch et al reported MT measurements of the human optic nerve using water-only images extracted with Dixon method in order to minimize the impact of meningeal fat signal (15). However, the latter approach might have limitations if intending to produce MT weighted images across a broad range of offset saturation frequencies. The accuracy of these measurements would be adversely impacted by the low SNR within images acquired at strong MT pre-saturation power. In our CSMT approach, the correction factor is calculated from only one fat/water separation measurement and can be applied to all the related MTR measurements at different saturation offset frequencies. Our method should be less affected by low SNR conditions during strong MT saturation and can readily generate the proton density weighted images required for MT measurements by adjust scanning parameters such as short TR and small flip angle.

Our study had several limitations. First, the CSMT method was tested using oil/agar phantoms, though one animal model study was performed to demonstrate feasibility of the method. However, for in vivo applications particularly in clinical settings, multiple confounding factors, including $\mathrm{B}_{0}$ and $\mathrm{B}_{1}$ inhomogeneity (30-32), $\mathrm{T}_{1}$ effects (33), eddy current effects (34), and susceptibility induced fat-resonance shifts (35), need to be considered. The effect of $\mathrm{B}_{0}$ inhomogeneity, as one of the potential limitations of the current method, should be included in the model, particularly for potential applications to abdominal imaging of visceral organs. Also, for these initial feasibility studies we considered only a single resonant frequency for fat signals but additional studies to characterize of the impact of multiple fat signal resonant peaks (36) is clearly warranted. Therefore, further translational studies for in vivo validation will be necessary. Second, with low water fraction (high fat content) within a voxel, inaccurate water fraction estimation may lead to relatively high errors in MTR $_{\text {true }}$ estimations (as indicated in the pure oil phantom in Fig. 2e and f). For in vivo applications it may be necessary to consider evaluation of only those voxels above a threshold water fraction level to avoid inaccuracies and associated misinterpretation. Third, recent studies have demonstrated the potential to use fat saturation pulses less 
sensitive to $\mathrm{B}_{0}$ and $\mathrm{B}_{1}$ heterogeneity to produce fat suppressed MT contrast images $(37,38)$. Additional future studies will be valuable for rigorous comparison of these fat-suppression based MT methods to our proposed CSMT approaches for quantitative MTR measurements. One additional limitation was that MT weighted images and fat/water separation images were acquired using two separate sequences. Integration of MT and fat/water separation within a single MRI sequence may be critical during clinical applications to both reduce scan times as well as avoid any misregistration that might occur due to motion between serial measurements.

In conclusion, we demonstrated the mechanism how fat fraction and TE can significantly impact observed MTR measurements and validated the feasibility of a new CSMT approach to eliminate the effects of fat upon MTR measurements. The CSMT technique could potentially be applied to provide more accurate MTR measurements in disease settings that involve both alterations in fat and macromolecular tissue content.

\section{Supplementary Material}

Refer to Web version on PubMed Central for supplementary material.

\section{Acknowledgments}

This publication has been funded in part by Grant Number CA181658 and CA159178 from the National Cancer Institute (NCI).

\section{References}

1. Wolff SD, Balaban RS. Magnetization transfer contrast (MTC) and tissue water proton relaxation in vivo. Magn Reson Med. 1989; 10(1):135-144. [PubMed: 2547135]

2. Ge Y, Grossman RI, Udupa JK, Babb JS, Kolson DL, McGowan JC. Magnetization transfer ratio histogram analysis of gray matter in relapsing-remitting multiple sclerosis. AJNR Am J Neuroradiol. 2001; 22(3):470-475. [PubMed: 11237968]

3. Bigot C, Vanhoutte G, Verhoye M, Van der Linden A. Magnetization transfer contrast imaging reveals amyloid pathology in Alzheimer's disease transgenic mice. Neuroimage. 2014; 87:111-119. [PubMed: 24188815]

4. Li W, Zhang Z, Nicolai J, Yang GY, Omary RA, Larson AC. Quantitative magnetization transfer MRI of desmoplasia in pancreatic ductal adenocarcinoma xenografts. NMR Biomed. 2013; 26(12): 1688-1695. [PubMed: 23940016]

5. Adler J, Swanson SD, Schmiedlin-Ren P, Higgins PDR, Golembeski CP, Polydorides AD, McKenna BJ, Hussain HK, Verrot TM, Zimmermann EM. Magnetization Transfer Helps Detect Intestinal Fibrosis in an Animal Model of Crohn Disease. Radiology. 2011; 259(1):127-135. [PubMed: 21324841]

6. Rosenkrantz AB, Storey P, Gilet AG, Niver BE, Babb JS, Hajdu CH, Lee VS. Magnetization transfer contrast-prepared MR imaging of the liver: inability to distinguish healthy from cirrhotic liver. Radiology. 2012; 262(1):136-143. [PubMed: 22114240]

7. Kim H, Booth CJ, Pinus AB, Chen P, Lee A, Qiu M, Whitlock M, Murphy PS, Constable RT. Induced hepatic fibrosis in rats: hepatic steatosis, macromolecule content, perfusion parameters, and their correlations--preliminary MR imaging in rats. Radiology. 2008; 247(3):696-705. [PubMed: 18403622]

8. Aisen AM, Doi K, Swanson SD. Detection of liver fibrosis with magnetic cross-relaxation. Magn Reson Med. 1994; 31(5):551-556. [PubMed: 8015410] 
9. Haase A, Frahm J, Hanicke W, Matthaei D. 1 H NMR chemical shift selective (CHESS) imaging. Physics in Medicine and Biology. 1985; 30(4):341. [PubMed: 4001160]

10. Frahm J, Haase A, Hanicke W, Matthaei D, Bomsdorf H, Helzel T. Chemical shift selective MR imaging using a whole-body magnet. Radiology. 1985; 156(2):441-444. [PubMed: 4011907]

11. Chen JH, Sambol EB, Decarolis P, O'Connor R, Geha RC, Wu YV, Singer S. High-resolution MAS NMR spectroscopy detection of the spin magnetization exchange by cross-relaxation and chemical exchange in intact cell lines and human tissue specimens. Magn Reson Med. 2006; 55(6):12461256. [PubMed: 16676334]

12. Chen JH, Le HC, Koutcher JA, Singer S. Fat-free MRI based on magnetization exchange. Magn Reson Med. 2010; 63(3):713-718. [PubMed: 20146235]

13. Lilburn, D., Cooper, A., Murphy, P., Sinclair, C., Semple, S., Janiczek, R. Initial experience using Magnetization Transfer with Iterative Decomposition of water and fat with Echo Asymmetry and Least-squares estimation (MT-IDEAL) in the abdomen. Proceedings of the 23th Annual Meeting of ISMRM; Toronto, Ontario, Canada. 2015. p. 1744

14. Holmes, J., Johnson, V., Hernando, D., Reeder, S., Samsonov, A. Magnetization transfer ratio (MTR) imaging in the presence of fat. Proceedings of the 23th Annual Meeting of ISMRM; Toronto, Ontario, Canada. 2015. p. 3365

15. Smith, AK., Dortch, RD., Smith, SA. Novel Quantitative Magnetization Transfer (qMT) of the Human Optic Nerve in vivo. Proceedings of the 22th Annual Meeting of ISMRM; Milan, Italy. 2014. p. 3329

16. Rinella ME. Nonalcoholic fatty liver disease: a systematic review. Jama. 2015; 313(22):22632273. [PubMed: 26057287]

17. Drukker BH, deMendonca WC. Fibrocystic change and fibrocystic disease of the breast. Obstet Gynecol Clin North Am. 1987; 14(3):685-702. [PubMed: 3323971]

18. Kredel LI, Batra A, Stroh T, Kuhl AA, Zeitz M, Erben U, Siegmund B. Adipokines from local fat cells shape the macrophage compartment of the creeping fat in Crohn's disease. Gut. 2013; 62(6): 852-862. [PubMed: 22543156]

19. Sam S. Adiposity and metabolic dysfunction in polycystic ovary syndrome. Horm Mol Biol Clin Investig. 2015; 21(2):107-116.

20. Reeder SB, Wen Z, Yu H, Pineda AR, Gold GE, Markl M, Pelc NJ. Multicoil Dixon chemical species separation with an iterative least-squares estimation method. Magn Reson Med. 2004; 51(1):35-45. [PubMed: 14705043]

21. Hu HH, Bornert P, Hernando D, Kellman P, Ma J, Reeder S, Sirlin C. ISMRM workshop on fatwater separation: insights, applications and progress in MRI. Magn Reson Med. 2012; 68(2):378388. [PubMed: 22693111]

22. Reeder, S., Hines, C., Yu, H., McKenzie, C., Brittain, J. On The Definition of Fat-Fraction for In Vivo Fat Quantification with Magnetic Resonance Imaging. Proceedings of the 17th Annual Meeting of ISMRM; Honolulu, Hawaii, USA. 2009. p. 211

23. Henkelman RM, Huang X, Xiang QS, Stanisz GJ, Swanson SD, Bronskill MJ. Quantitative interpretation of magnetization transfer. Magn Reson Med. 1993; 29(6):759-766. [PubMed: 8350718]

24. Zhong X, Nickel MD, Kannengiesser SA, Dale BM, Kiefer B, Bashir MR. Liver fat quantification using a multi-step adaptive fitting approach with multi-echo GRE imaging. Magn Reson Med. 2014; 72(5):1353-1365. [PubMed: 24323332]

25. Li W, Zhang Z, Nicolai J, Yang GY, Omary RA, Larson AC. Magnetization transfer MRI in pancreatic cancer xenograft models. Magn Reson Med. 2012; 68(4):1291-1297. [PubMed: 22213176]

26. Li W, Zhang Z, Li K, Jin N, Zhang Y, Zhang T, Miller FH, Larson AC. Respiratory self-gating for free-breathing magnetization transfer MRI of the abdomen. Magn Reson Med. 2015; 73(6):22492254. [PubMed: 24962475]

27. Chen JH, Yeung HN, Lee SK, Chai JW. Evaluation of liver diseases via MTC and contrast agent. J Magn Reson Imaging. 1999; 9(2):257-265. [PubMed: 10077022] 
28. Guo J, Erickson R, Trouard T, Galons JP, Gillies R. Magnetization transfer contrast imaging in Niemann pick type C mouse liver. J Magn Reson Imaging. 2003; 18(3):321-327. [PubMed: 12938127]

29. Miese F, Kircheis G, Wittsack HJ, Wenserski F, Hemker J, Modder U, Haussinger D, Cohnen M. 1H-MR spectroscopy, magnetization transfer, and diffusion-weighted imaging in alcoholic and nonalcoholic patients with cirrhosis with hepatic encephalopathy. AJNR Am J Neuroradiol. 2006; 27(5):1019-1026. [PubMed: 16687536]

30. Hernando D, Haldar JP, Sutton BP, Ma J, Kellman P, Liang ZP. Joint estimation of water/fat images and field inhomogeneity map. Magn Reson Med. 2008; 59(3):571-580. [PubMed: 18306409]

31. Sinclair CD, Morrow JM, Hanna MG, Reilly MM, Yousry TA, Golay X, Thornton JS. Correcting radiofrequency inhomogeneity effects in skeletal muscle magnetisation transfer maps. NMR Biomed. 2012; 25(2):262-270. [PubMed: 21796708]

32. Dortch RD, Dethrage LM, Gore JC, Smith SA, Li J. Proximal nerve magnetization transfer MRI relates to disability in Charcot-Marie-Tooth diseases. Neurology. 2014; 83(17):1545-1553. [PubMed: 25253751]

33. Liu CY, McKenzie CA, Yu H, Brittain JH, Reeder SB. Fat quantification with IDEAL gradient echo imaging: correction of bias from T(1) and noise. Magn Reson Med. 2007; 58(2):354-364. [PubMed: 17654578]

34. Hernando D, Hines CD, Yu H, Reeder SB. Addressing phase errors in fat-water imaging using a mixed magnitude/complex fitting method. Magn Reson Med. 2012; 67(3):638-644. [PubMed: 21713978]

35. Karampinos DC, Yu H, Shimakawa A, Link TM, Majumdar S. Chemical shift-based water/fat separation in the presence of susceptibility-induced fat resonance shift. Magn Reson Med. 2012; 68(5):1495-1505. [PubMed: 22247024]

36. Yu H, Shimakawa A, McKenzie CA, Brodsky E, Brittain JH, Reeder SB. Multiecho water-fat separation and simultaneous R2* estimation with multifrequency fat spectrum modeling. Magn Reson Med. 2008; 60(5):1122-1134. [PubMed: 18956464]

37. Abe T. Fast fat suppression RF pulse train with insensitivity to B1 inhomogeneity for body imaging. Magn Reson Med. 2012; 67(2):464-469. [PubMed: 21633968]

38. Zhao F, Nielsen JF, Swanson SD, Fessler JA, Noll DC. Simultaneous fat saturation and magnetization transfer contrast imaging with steady-state incoherent sequences. Magn Reson Med. 2015; 74(3):739-746. [PubMed: 25252173] 
A

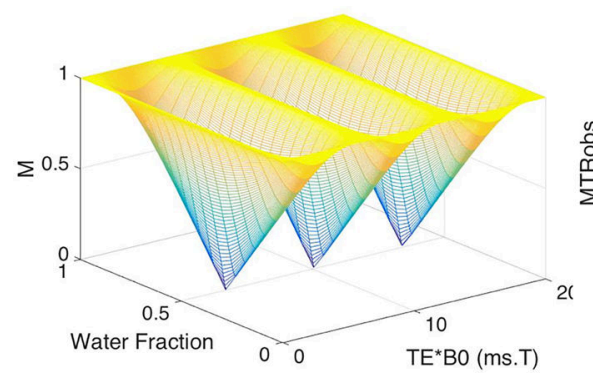

B

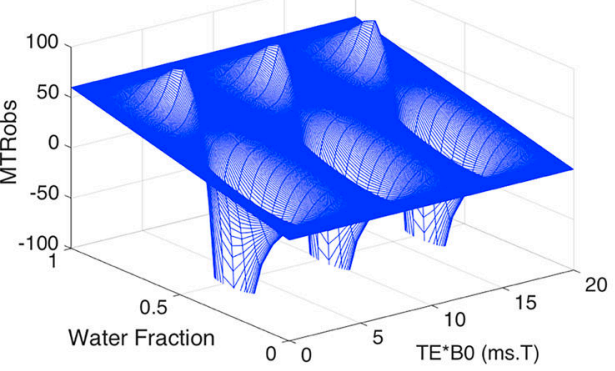

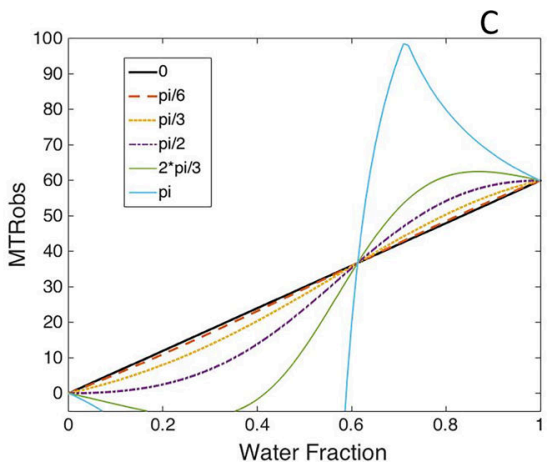

Fig. 1.

MRI signal (M) without MT saturation and MTR $_{\mathrm{obs}}$ for a voxel with presence of fat, free water and macromolecules showing the effects of different values of water fraction as well as the product of echo time and field strength $\left(\mathrm{TE} \bullet \mathrm{B}_{0}\right)$. The variations of the water fraction and $\mathrm{TE} \cdot \mathrm{B}_{0}$ (a) lead to changes in the MRI signal (M) (a) and $\mathrm{MTR}_{\mathrm{obs}} \mathrm{b}$ ). $\mathrm{MTR}_{\mathrm{obs}}$ for water and fat signals with phase differences ranging from in-phase to completely out of phase, across a broad range of water fraction values (c). A linear relationship of $\mathrm{MTR}_{\mathrm{obs}}$ with water fraction is shown when fat and water are in-phase (black line). The remaining parameters used in this simulation include: $\mathrm{M}_{\mathrm{a}}=1, \mathrm{M}_{\mathrm{satw}}=0.4$, chemical shift between fat and water $=$ $3.5 \mathrm{ppm}$. 

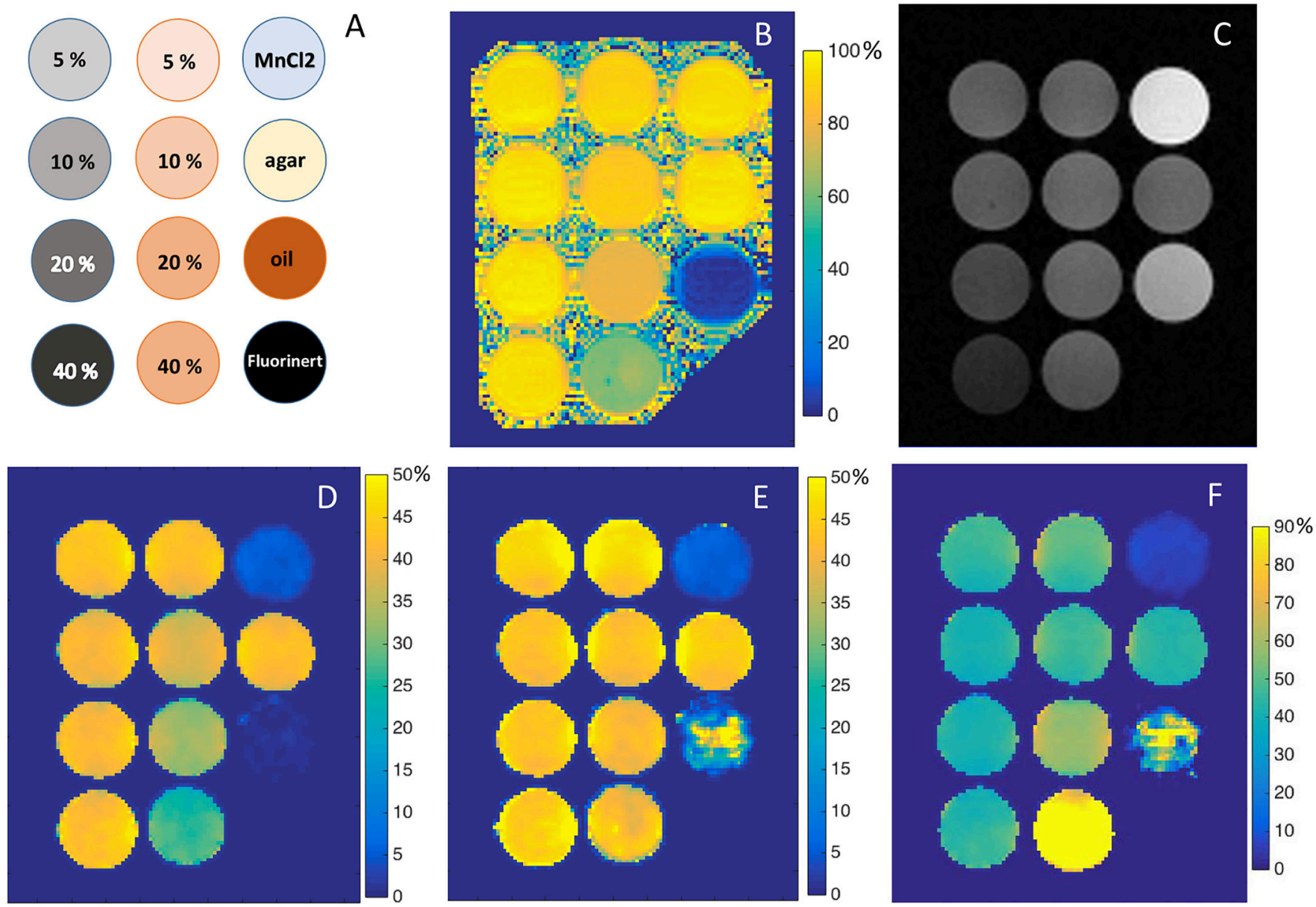

Fig. 2.

Phantom setup (A), water fraction map (B), representative MT weighted images with water and fat signals in phase (C), corresponding $\mathrm{MTR}_{\text {obs }}$ maps (D), calculated MTR true map (E), and MTR $_{\text {true }}$ maps with water and fat signals in opposed phase $(F)$. MT saturation pulse has a FA of $800^{\circ}$, length of $10 \mathrm{~ms}$, and off-resonance frequency of $1 \mathrm{kHz}$; TE $=2.24 \mathrm{~ms}$ in $(\mathrm{C})$, and $\mathrm{TE}=3.36 \mathrm{~ms}$ in $(\mathrm{F})$; Unit for the color bars: percent $(\%)$ 

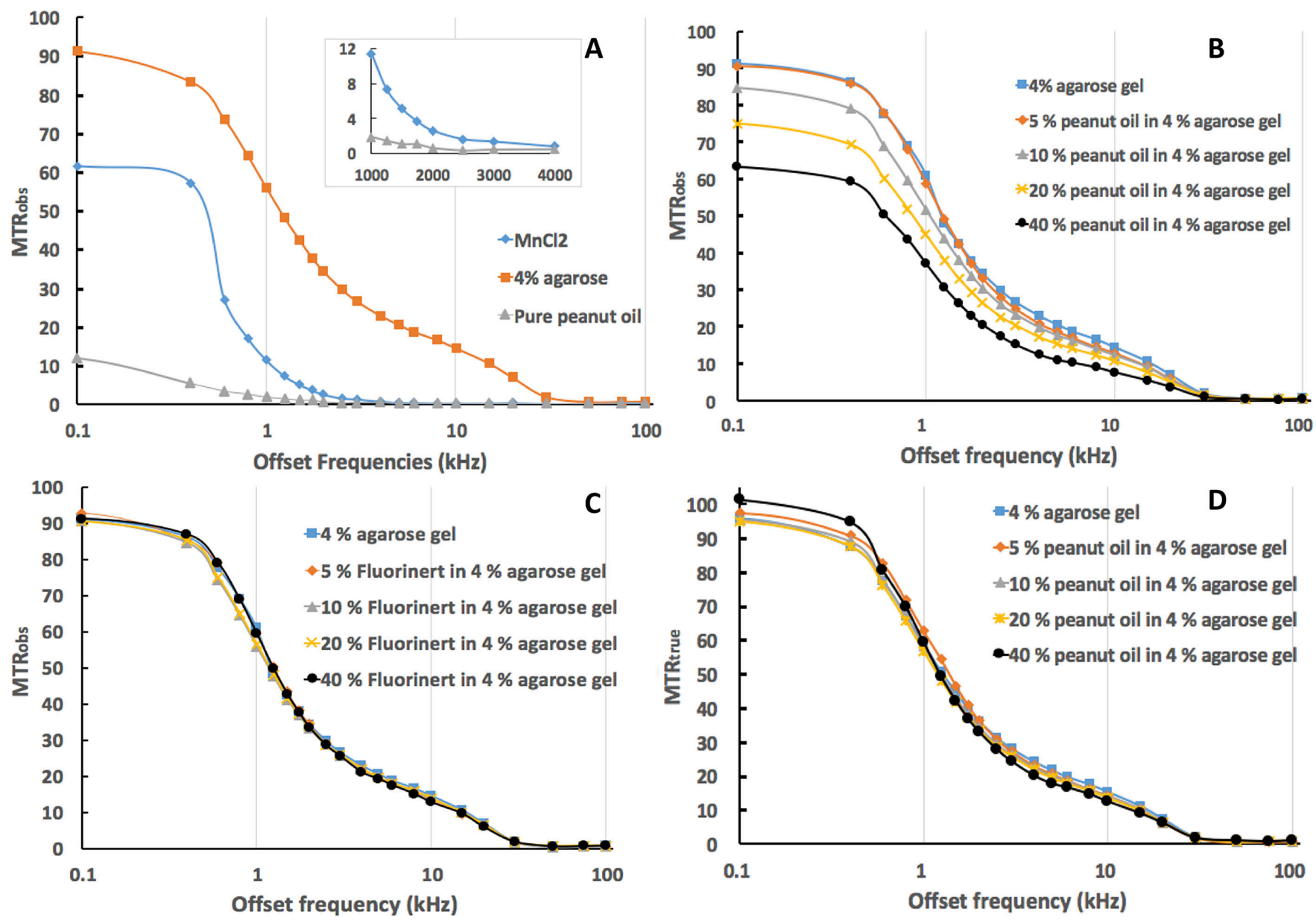

Fig. 3.

Graphs of MTRs at various MT offset frequencies showing the direct saturation effect MT saturation on peanut oil and $\mathrm{MnCl}_{2}$ (A), $\mathrm{MTR}_{\mathrm{obs}}$ of oil/agar phantoms (B), MTR $\mathrm{obs}_{\text {of }}$ fluorocarbon/agar phantoms (C), and MTR true of oil/agar phantoms. (A) validates that the oil phantom was much less affected by direct saturation of the MT pulse compared to the conventional control phantom ( $\mathrm{MnCl}_{2}$ solution) and tissue simulation phantom (4\% agarose gel). B, C, and D demonstrate that our method generated comparable MTRs for the oil/agar phantom, comparable to control phantoms that had similar macromolecule content but lacked oil component. MT saturation pulse has a FA of $800^{\circ}$, length of $10 \mathrm{~ms}$. 


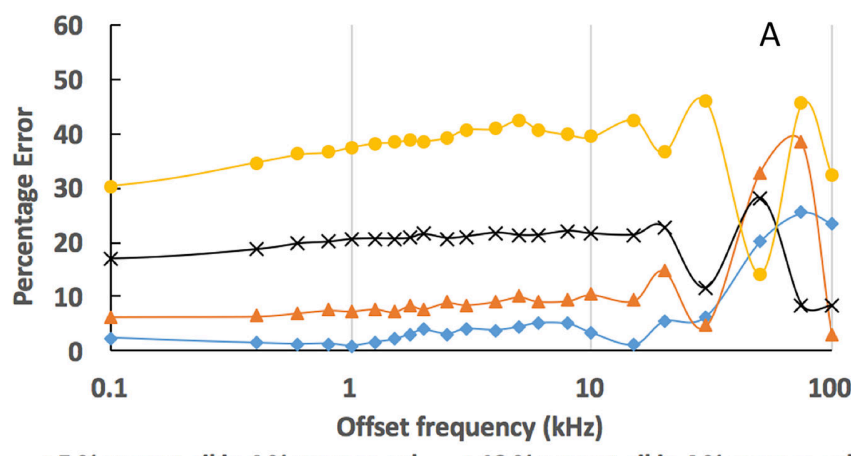

$\rightarrow 5 \%$ peanut oil in $4 \%$ agarose gel $\$ 10 \%$ peanut oil in $4 \%$ agarose gel $* 20 \%$ peanut oil in $4 \%$ agarose gel $-40 \%$ peanut oil in $4 \%$ agarose gel

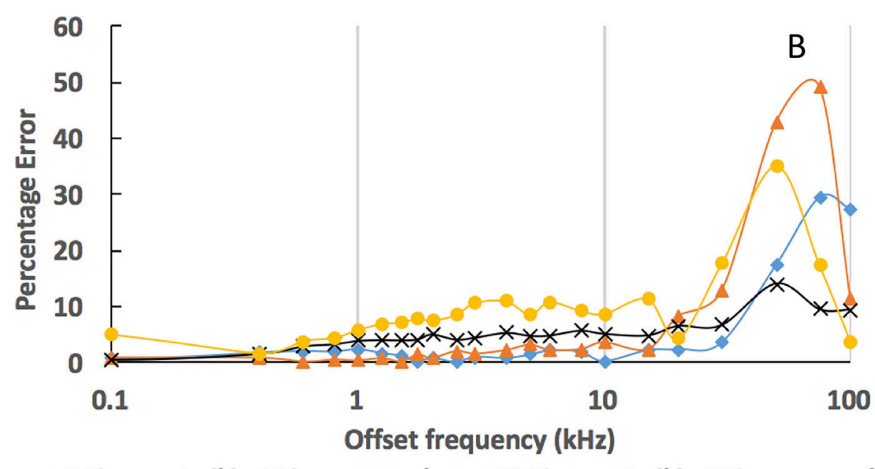

$\rightarrow 5 \%$ peanut oil in $4 \%$ agarose gel $₫-10 \%$ peanut oil in $4 \%$ agarose gel *20\% peanut oil in $4 \%$ agarose gel $-40 \%$ peanut oil in $4 \%$ agarose gel

Fig. 4.

Graphs showing the percentage errors of $\mathrm{MTR}_{\mathrm{obs}}$ and $\mathrm{MTR}_{\text {true }}$ measurements at various MT offset frequencies when comparing to corresponding fluorocarbon/agar phantoms. A. the percentage errors between $\mathrm{MTR}_{\mathrm{obs}}$ and $\mathrm{MTR}_{\text {ref }}$; $\mathrm{B}$. the percentage errors between $\mathrm{MTR}_{\text {true }}$ and $\mathrm{MTR}_{\text {ref. }}$ MT saturation pulse has a FA of $800^{\circ}$, length of $10 \mathrm{~ms}$. 

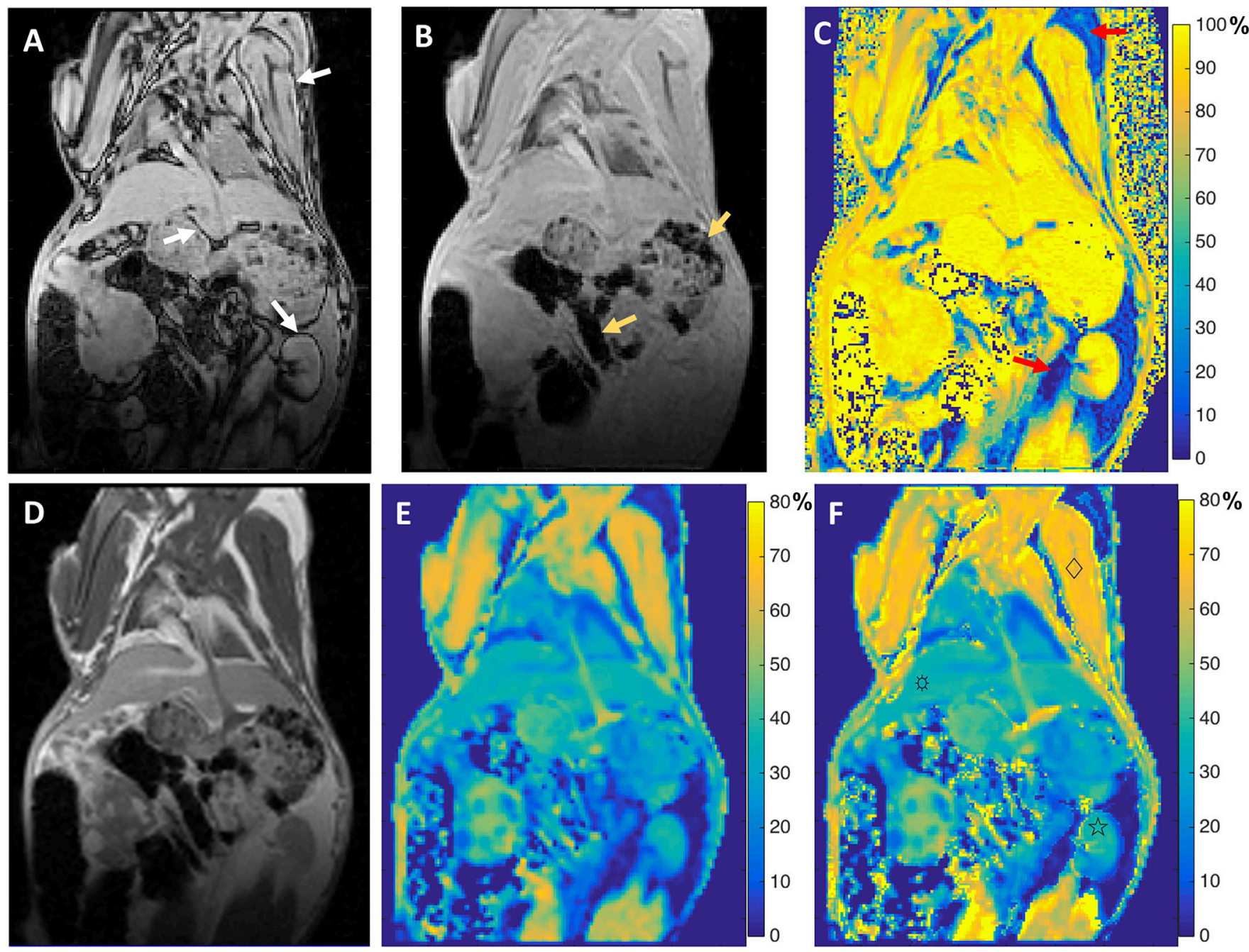

Fig. 5.

Images acquired on a rabbit. A. Opposed-phase image with TE $=1.19 \mathrm{~ms}$; B. in-phase image with $\mathrm{TE}=2.46 \mathrm{~ms} ; \mathrm{C}$. corresponding water fraction map; D. MT weighted images with water and fat signals in phase (TE $=2.24 \mathrm{~ms}$ ); E: corresponding $\mathrm{MTR}_{\mathrm{obs}}$ map; and $\mathrm{E}$. calculated MTR $_{\text {true }}$ map. Slight increases in calculated MTR values were observed for skeletal muscle $(\diamond)$, liver ( $)$, and kidney ( and $37.36 \pm 1.23, \mathrm{MTR}_{\text {true }}$ values of $66.44 \pm 2.73,36.60 \pm 1.06$, and $38.35 \pm 1.38$ respectively). 